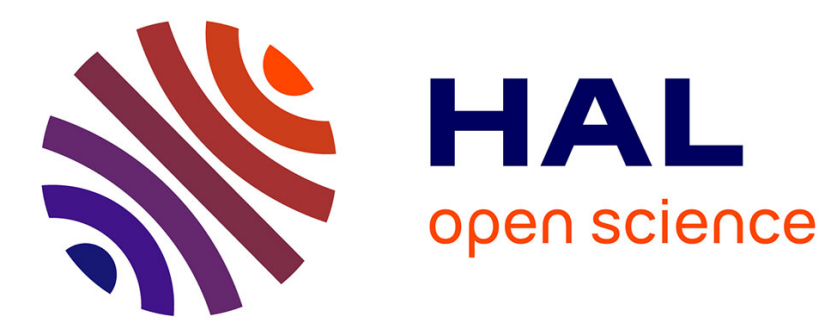

\title{
A Dielectric Low-Perturbation Field Scanner for Multi-Path Environments
}

Andréa Cozza, Francesco Masciovecchio, Cécile Dorgan, Mohamed Serhir, Florian Monsef, Dominique Lecointe

\section{- To cite this version:}

Andréa Cozza, Francesco Masciovecchio, Cécile Dorgan, Mohamed Serhir, Florian Monsef, et al.. A Dielectric Low-Perturbation Field Scanner for Multi-Path Environments. IEEE Transactions on Antennas and Propagation, 2017, 65 (4), pp.1978 - 1987. 10.1109/TAP.2017.2670563 . hal-01445476

\section{HAL Id: hal-01445476}

https://hal-centralesupelec.archives-ouvertes.fr/hal-01445476

Submitted on 24 Jan 2017

HAL is a multi-disciplinary open access archive for the deposit and dissemination of scientific research documents, whether they are published or not. The documents may come from teaching and research institutions in France or abroad, or from public or private research centers.
L'archive ouverte pluridisciplinaire $\mathbf{H A L}$, est destinée au dépôt et à la diffusion de documents scientifiques de niveau recherche, publiés ou non, émanant des établissements d'enseignement et de recherche français ou étrangers, des laboratoires publics ou privés. 


\title{
A Dielectric Low-Perturbation Field Scanner for Multi-Path Environments
}

\author{
Andrea Cozza, Senior Member, IEEE, Francesco Masciovecchio, Cécile Dorgan, Mohamed Serhir, Member, IEEE, \\ Florian Monsef, Member, IEEE, Dominique Lecointe
}

\begin{abstract}
Well-established solutions capable of scanning electromagnetic fields in anechoic environments essentially rely on the massive use of absorbers, in order to keep under check energy scattered by the metallic parts of an automatic positioning system. Solutions of this kind are convenient whenever the field to scan is the result of line-of-sight (LOS) propagation. In complex media, where multi-path propagation imply non-LOS contributions, the introduction of absorbing materials can irremediably alter the behavior of the medium under test and therefore the field to be scanned. In this case, a field scanner rather needs to be as transparent as possible. This paper introduces the main ideas behind an alternative automatic positioning system designed in order to ensure a low perturbation of the response of a medium. The impact of the scanner is assessed when used in a reverberation chamber with a varying degree of reverberation, in order to emulate the conditions found in realistic complex media. Expected applications are mainly found in sounding propagation in complex media.
\end{abstract}

Index Terms-Complex media, multi-path propagation, field scanner, perturbation analysis.

\section{INTRODUCTION}

A robotic field scanner must fulfill a number of constraints: it needs to be stiff enough as to maintain its nominal shape while moving potentially heavy antennas/probes, allow their fast displacement with sufficient accuracy in their final position and not perturb the field distribution it is meant to measure. The standard solution to all these constraints is to rely on metallic structures, typically made of steel or aluminium, because of their stiffness due to Young elastic moduli in the tens of GPa, low manufacturing costs and high versatility, plus a large choice of standard beam profiles. When used in anechoic environments, the high reflectivity of metallic surfaces is kept in check by covering them with electromagnetic absorbing materials, typically dissipative foams [1], [2].

Consider then the case of complex media, where waves propagate along a broad spectrum of directions, potentially with multiple interactions back and forth along a medium, as schematically illustrated in Fig. 1. Such configurations are widely found in practical situations, e.g., in indoor and urban propagation, and applies as well to most industrial sites. If

Part of the work here reported was funded by the French National Research Agency through the grant ANR-12-ASTR-0005, MIMOCHIC project, within the framework of the 2012 ASTRID program. The design and manufacturing of the robot were funded by Digiteo, under the AUTOTREC project.

A. Cozza, M. Serhir, F. Monsef and D. Lecointe are with PIEM, GeePs (UMR 8507), 11 rue Joliot-Curie, 91192 Gif-sur-Yvette (France). Francesco Masciovecchio was with Politocenico di Milano, Milano (Italy) at the time of the tests; he currently is with ESE Engineering Services for Energy S.r.1., Milano (Italy). Cécile Dorgan is with DGA Techniques Aéronautiques, Balma (France). Contact e-mail: andrea.cozza@ieee.org.

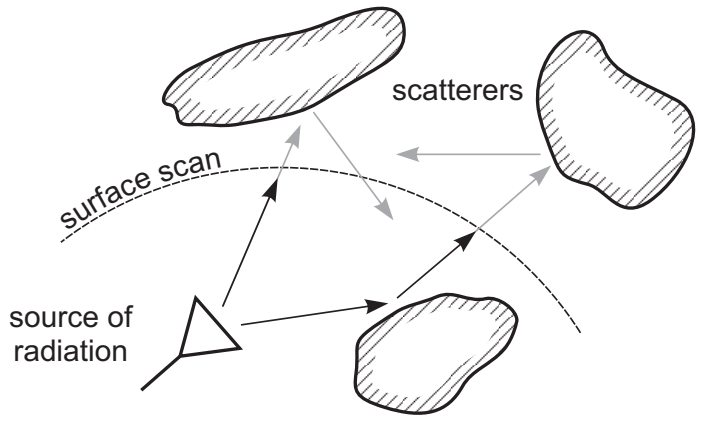

Fig. 1: Schematic representation of propagation in a complex medium, where an absorbed-covered surface is scanned. Grey lines stand for propagation paths that would be affected by the presence of absorbers over the scanned surface. Only line-ofsight contributions (black lines) are unmodified.

field distributions in these environments were measured over a surface by means of standard scanners, the presence of absorbers could alter the propagation of waves as found in the original medium. In the example in Fig. 1, energy along the greyed paths would be attenuated, leading to a reduced set of propagation paths and ultimately to what would appear as a simpler medium. Even worse, as the position of the scanner changes, the perturbation of the medium would also change, leading with non-consistent information.

Wireless propagation in complex media are seldom tested in situ, since it is in general costly to do so. Most often, reverberation chambers are used as test facilities. A large literature has been building up during the last ten years, showing that they are capable of reproducing similar statistical conditions as in real-life media [3]-[5]. While statistical descriptions of propagation within these media are now well established, there is a clear lack of thorough experimental data about wave propagation, making very difficult to validate these models. Typically, only figures of merit involving a few scattered points within a reverberation chamber are considered. In order to extract enough information about propagation paths inevitably amounts to cumbersome experimental procedures, involving the manual displacement of probes on a large number of positions [6]. The comparison with anechoic chambers is striking, where full surfaces can be automatically scanned for accurate field samples.

In order to avoid perturbing the propagation of waves in complex media, a field scanner should be weakly scatter- 


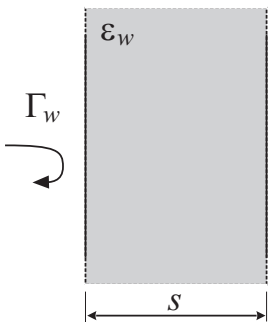

(a)

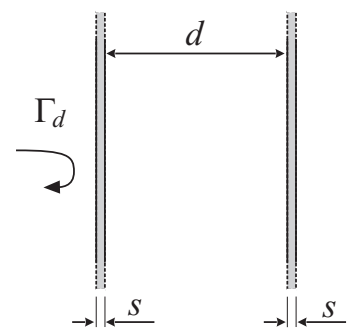

(b)
Fig. 2: Thin-plate models for the computation of reflection coefficients: (a) single thin wall and (b) double wall.

ing, and also ideally lossless. Keeping aside for a moment the issues of mechanical transmission and motors, a lowperturbation robot could be made out of materials as transparent as possible to radio-frequency waves. Typically, this would be possible only when using foams, such as styrofoam or Rohacell, with $\epsilon_{r} \lesssim 1.2$, and certain polymers such as PTFE, with $\epsilon_{r} \simeq 2$. Unfortunately, all of these materials offer too weak Young elastic moduli, thus low stiffness: typical values for bulk polymers are in the order of a few GPa, yielding by far lower values once expanded into foams. They also have low fracture strength, i.e., break easily. Their relatively rough surfaces make them intrinsically subject to wear, e.g., due to repetitive friction as a probe moves over them. Moreover, manufacturing processes are usually based on (subtractive) bulk machining techniques, as foams do not come in the shape of beams and can hardly be soldered, bolted together or bent.

The alternative solution we introduce in this paper is rather based on the use of non-transparent dielectric materials, namely composite materials presenting three features: 1) very weak Ohmic losses; 2) intermediate-range values of their relative dielectric permittivity, well above that of air, but smaller than 10, typically around 5; 3) a relatively high Young modulus. They can be cut from sheets and assembled in a number of different ways.

We first argue about the potential use of non-transparent materials for building a weakly perturbative robot in Sec. II while the main ideas behind its design are summarized in Sec. III. The low reflectivity enabled by the hollow structures employed in the robot is studied in Sec. IV. A thorough experimental assessment of the perturbation introduced in a reverberation chamber by a prototype of the scanner is presented in Sec. V and the main experimental results are discussed in Sec. VI.

The scanner is found to ensure a coherence about $90 \%$ in an unloaded reverberation chamber operated up to $1 \mathrm{GHz}$, characterized by an average spread time $\bar{\tau}=1.2 \mu \mathrm{s}$, implying a very rich multiple-path propagation. When, after introducing losses in the chamber leading to $\bar{\tau}=0.16 \mu \mathrm{s}$, and therefore strongly reducing the number of propagation paths, the same coherence can be maintained up to $6 \mathrm{GHz}$.

\section{Plane-WAVE REFLECTION FROM THIN-PLATE STRUCTURES}

In the rest of the paper we consider hollow dielectric structures and assess how strongly they perturb multi-path propagation media. The relationship existing between freespace scattering of the robot and the final perturbation of wave propagation in a complex media is far from obvious, as witnessed by decades of research on radiative transfer and multiple-scattering techniques [7], [8]. Moreover, it is by far simpler to track modifications in the propagation of waves in a complex medium (see Sec. V) than to measure the bistatic scattering response of an electrically large structure. For these reasons, the effect of the robot is directly tested experimentally in a multi-path environment, as it would in practical applications, without studying its free-space behavior as a scatterer.

Still, we deem necessary to understand under what conditions dielectric materials with $\epsilon_{r}>1$ can become weakly scattering structures. To this end, this section studies the apparent reflection coefficient of a set of two parallel thin dielectric plates. While this structure is clearly different from those used in the proposed field scanner, it is still capable of reproducing the main phenomenon behind the low scattering that the robot displays at low frequencies and the rapid increase observed at higher frequencies. In this respect, it must not be regarded as a design tool, but rather as a way of understanding what are the main parameters that control the level of reflectivity of the robot and thus its perturbations during measurements.

We consider a plane wave impinging orthogonally over an infinitely large dielectric plate of thickness $s \ll \lambda$, as in 2(a), where $\lambda$ is the wavelength in the background medium at the working frequency $\nu$. The reflection coefficient $\Gamma_{w}$ from a single wall can be computed by first introducing

$$
\Gamma_{o}=\left(\sqrt{\epsilon_{w}}-1\right) /\left(\sqrt{\epsilon_{w}}+1\right)
$$

the reflection coefficient when passing from one half-space,the background medium, here air, to another one with a relative dielectric permittivity $\epsilon_{w}$. For a plate, two such discontinuities appear, one when passing from the background medium to the inside of the plate, and then passing back to the background at the other side.

The overall reflection for the plate can be expanded into a sequence of single-event interactions of partial waves propagating back and forth through the wall [9]. For the solution discussed in Sec. III, only materials with $\epsilon_{w}<6$ are considered, as expected for composite materials based on glass fibers. In this case, $\Gamma_{o} \leq 0.42$. Hence, after the impinging wave crosses the left interface of the wall and is reflected by the right interface, the amplitude of the wave reflected again rightward will not account for more than $7 \%$ of the impinging amplitude.

We therefore take into account only the first two interactions, so that $\Gamma_{w}$ can be written as

$$
\Gamma_{w}=\Gamma_{o}\left(1-\mathrm{e}^{-2 \mathrm{j} \beta s}\right),
$$

which, for $s \ll \lambda$, can be approximated by

$$
\Gamma_{w} \simeq \mathrm{j} \omega 2 \Gamma_{o} s / c_{o}=\mathrm{j} 4 \pi \Gamma_{o} s / \lambda,
$$


with $c_{o}$ the speed of light in the background medium. This result shows that the frequency response of the plate is derivative, so that the higher the frequency, the stronger the reflection. Recalling our interest for weakly perturbative structures, the derivative behavior can be exploited in the lowfrequency range, in order to minimize the amount of power scattered by the structure, in spite of the use of materials with relative dielectric permittivities well higher than that of air, involving a $\left|\Gamma_{o}\right|$ not necessarily negligible with respect to one. A more detailed analysis of this kind of scattering can be found in [10], where all interactions and directions of arrival are taken in account.

Eq. (3) can now be used in order to study the reflectivity of a pair of thin walls, as in Fig. 2(b). This configuration is important since it can represent part of the cross-section of a hollow dielectric structure. Taking again into account only the first interactions with the walls, the overall reflectivity is now

$$
\Gamma_{d}=\Gamma_{w}\left[1+\left(1+\Gamma_{w}\right)^{2} \mathrm{e}^{-j 2 k_{o} d}\right] .
$$

The frequency evolution of (3) and (4) are used in Sec. IV as terms of comparison with numerical results of the local reflectivity of one robot arm. The results shown there suggest that although neglecting the presence of the lateral sides of the robot arm, (3) and (4) capture the essential phenomenon behind the frequency-dependent perturbation of the robot. We want to stress again that the actual figure of merit for perturbation is rather the coherence of the medium, which is discussed in Sec. V.

\section{GUIDELINES BEHIND THE SCANNER DESIGN}

The type of surface described by a scanner depends on the intended use of field data. Examples are well-known in nearfield antenna testing, where planar, cylindrical and spherical surfaces are standard choices. Here a spherical surface was chosen in order to ensure omnidirectional features. We deem this property fundamental when sounding a medium, especially a diffusive one where waves can in principle follow any direction of propagation. Given the metallic floor of the reverberation chamber, only $2 \pi$ steradian need to be covered, corresponding to a hemispherical surface. Waves propagating along other directions, corresponding to reflections over the floor, can be straightforwardly computed off-line.

The hemispherical surface $\Sigma$ is covered by moving a probe holder to an azimuthal angle $\varphi$ around the polar axis, and an elevation angle $\theta$. The polar axis lies parallel to the ground. Designing a low perturbation scanner requires that two conditions be met at the same time: reducing scattering from the scanner structure, e.g., its arms, and avoid moving metallic parts.

A hemispherical surface can be scanned by using a circular arm as a guiding structure for a probe holder, if the arm could rotate around the polar axis. The main problem with this kind of solution comes from the deflection of the arm, caused by gravity, when it lies close to horizontal, as in the case in Fig. 3(a). Because of the limited stiffness of composite materials, the direct consequence of deflection would be a systematic bias between the final position of the probe holder and the original command, potentially leading to a mechanical hysteresis.

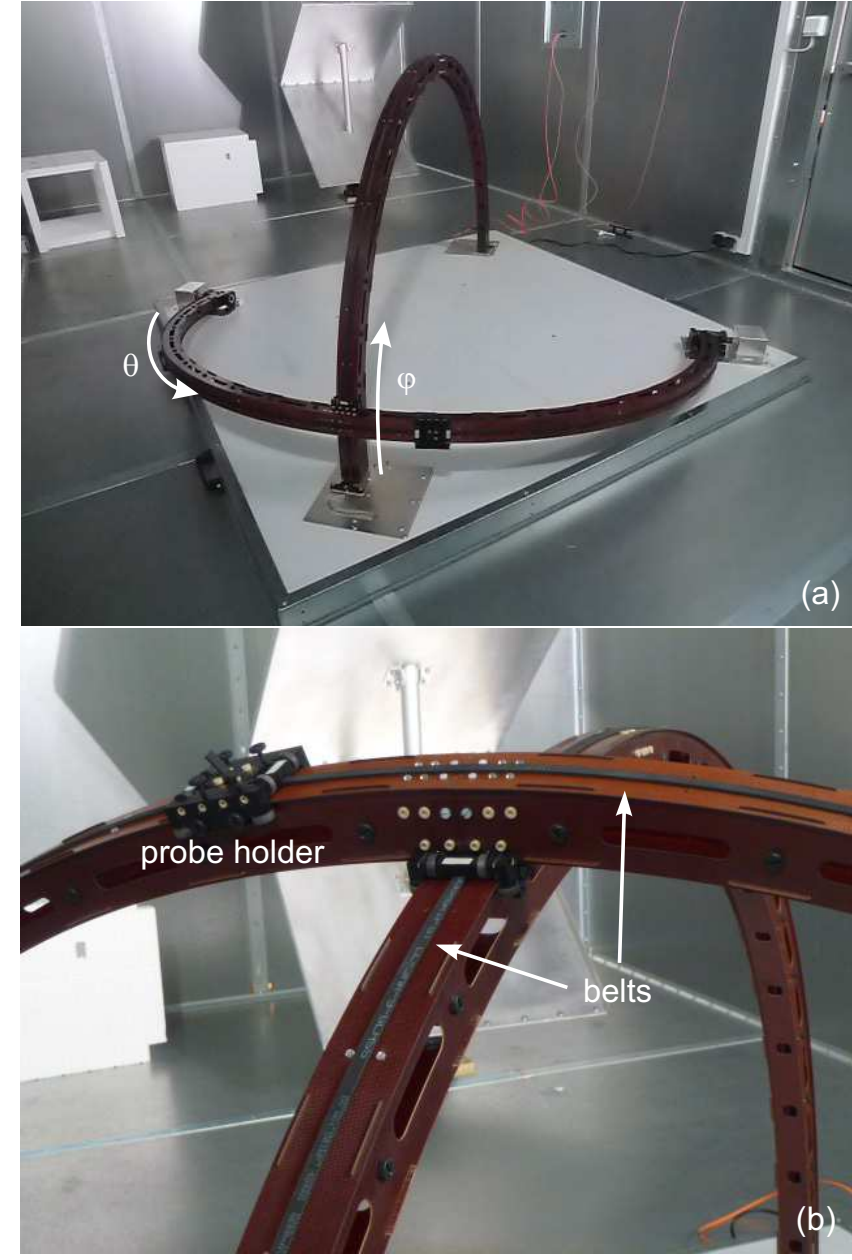

Fig. 3: The dielectric robot (a) with the two dielectric arms and its metallic platform hosting the motors and a close-up (b) of the arms crossing, showing the belts and probe holder.

Since the moving arm is a curved beam, there is no simple way of predicting its deflection as a function of its geometry and its constitutive materials. But an approximate understanding of how deflection can be controlled can be gained by considering the related case of a straight beam, fixed at its ends, where the effect of gravity is concentrated at its middle over a single point. In this case, the deflection $d$ is given by [11]

$$
d=\frac{W L^{3}}{48 E I},
$$

where $W$ is the weight of the beam, $L$ its length, $E$ Young elastic modulus of its constitutive material and $I$ the transversal inertia moment of the beam. Given the length of the beam, the main parameter available for controlling $d$ is the inertia moment; since $I$ is proportional to the square of the cross section, it can be effectively used for keeping in check the deflection. Eq. (5) shows why a high Young modulus is important, since $d$ is inversely proportional to it. Hence, switching from the $70 \mathrm{GPa}$ of aluminium to the average 30 $\mathrm{GPa}$ of glass-reinforced polymer matrices more than doubles the deflection. Clearly, since (5) is a crude model for a curved beam, it cannot be used for designing the arm, and 
numerical models would be necessary. A mechanical finite element analysis showed that for $|d|<1 \mathrm{~mm}$, the arm would need to be more than $10 \mathrm{~cm}$ wide and at least $5 \mathrm{~mm}$ thick, leading to potentially high wave scattering.

A more effective way of reducing the deflection without overdesigning the moving arm is to introduce a second hemispherical arm, now fixed, as shown in Fig. 3; the moving arm is now no longer rotated by motors at its base, but rather by pulling a belt fixed at the center of the mobile arm and sliding along the upper surface of the fixed arm. Two advantages come with this choice : 1) the moving arm is now supported by a new fixed point halfway its total length, with (5) roughly implying an eightfold reduction of the deflection; 2 ) the moving arm is now put into rotation by a direct pull at its extremal point, rather than from its base. Less torque can be used in this configuration, also reducing the strain at the ends of the moving arm. These observations result in thinner arms, $4 \mathrm{~cm}$ wide, hollow with walls $1 \mathrm{~mm}$ thick, capable of ensuring a maximum deflection below $1 \mathrm{~mm}$ at any position for a probe holder up to $200 \mathrm{~g}$. Fig. 3(b) shows some details of the mobile arm manufactured for our prototype robot. Openings were cut throughout the lateral surfaces in order to further reduce its level of scattering.

Reducing scattering from the arms is only part of the problem. The second issue to solve is the perturbation potentially generated by moving metallic parts. As long as they do not modify the boundary conditions of the medium, their effect can be neglected, e.g., in case of a metallic floor. But if they were found along mechanical transmission chains, e.g., as part of the probe holder, their effect would be to enforce new boundary conditions. For this reason, we chose to integrate the motors into a metallic platform over which the robot is mounted.

Movements along the $\varphi$ and $\theta$ directions are imparted by means of glass-reinforced toothed belts, driven by pairs of coupled (synchronized) motors in a master-slave configuration; the motors are controlled by a microprocessor integrated into the pedestal, communicating with a computer through an optical-fiber link. Two belts are visible in Fig. 3(b), sliding over the top surface of each arm. The two motors responsible for movements along $\varphi$ are effectively integrated into the scanner platform, while those for the $\theta$ movements are found in metallic boxes about $10 \mathrm{~cm}$ high, as seen in Fig. 3(a). The presence of these boxes makes it impossible to reach positions closer than 5 degrees to the polar axis of the scanner.

The probe holder, made of HDPE (high-density polyethylene), can host electro-optical probes, which are inherently very weakly scattering. They were not included during the tests, in order to enable faster displacements.

A detailed description of the prototype designed out of these guidelines and tested in Sec. V can be found in [12].

\section{ASSESSING THE REFLECTIVITY FROM A ROBOT ARM}

Having discussed the reasons that led to choosing a hollow cross-section $4 \mathrm{~cm}$ wide, we can now assess its effective reflectivity and compare it with (3) and (4) from Sec. II. CST's Microwave Studio was used for this purpose, modeling the mobile arm of the robot, described in Sec. III. The simulations

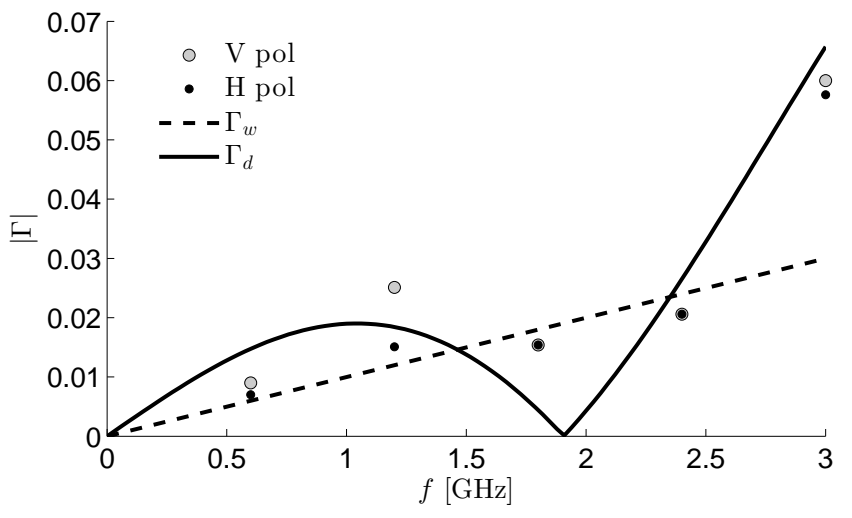

Fig. 4: Reflection coefficient of the mobile arm of the robot, for a plane wave impinging orthogonally to the plane containing the arm, for two polarizations. The two theoretical curves refer to the models in (3) and (4), for the configurations in Fig. 2. See Sec. IV for details about the way the reflection coefficients were estimated from numerical simulations.

consisted in having a linearly polarized plane wave impinging on the mobile arm of the robot and assessing the degree of reflectivity of the arm. Only the mobile arm was tested, since these simulations are only meant to check whether (3) and (4) correctly identify the physical mechanisms behind the frequency-dependent scattering from the hollow structures constituting the robot.

Clearly, the scattering from a robot arm is a function of the direction of arrival of an incoming plane wave. Only one direction of arrival was studied, the one leading to the strongest scattering, which can be expected for a plane wave with its front parallel to the arm planar lateral surface. In this case all of the local contributions over its surface are in phase with one another producing the strongest back scattering.

Simulations were run to cover frequencies up to $3 \mathrm{GHz}$. The large ratio between the maximum dimensions of the structure $(2 \mathrm{~m})$ and its finest details (about $1 \mathrm{~mm}$ ) made it impossible to run simulations at higher frequencies. The total field distribution excited by the impinging plane waves was sampled close to the external surface of the robot, at less than $\lambda$, in order to reduce the impact of propagation divergence, which could lead to conclude a lower level of scattering, since the scattered wave attenuates as it fans out in space. The local reflectivity of the arm was then extracted by computing the standing wave ratio from the total field samples and converting it back into a reflection coefficient, which measures the local reflectivity of the robot arm. This operation was repeated for vertical and horizontally polarized plane waves.

The results are show in Fig. 4, where they are compared to the reflectivity estimated by the models introduced in Sec. II, in particular by (4). The theoretical and numerical results compare favorably, implying that the main mechanism behind the arm scattering is the reflection from the thin walls composing the hollow structure of each arm. As the frequency increases, the contribution from the opposed thin wall increases the overall scattering; in the case considered in these tests, the 


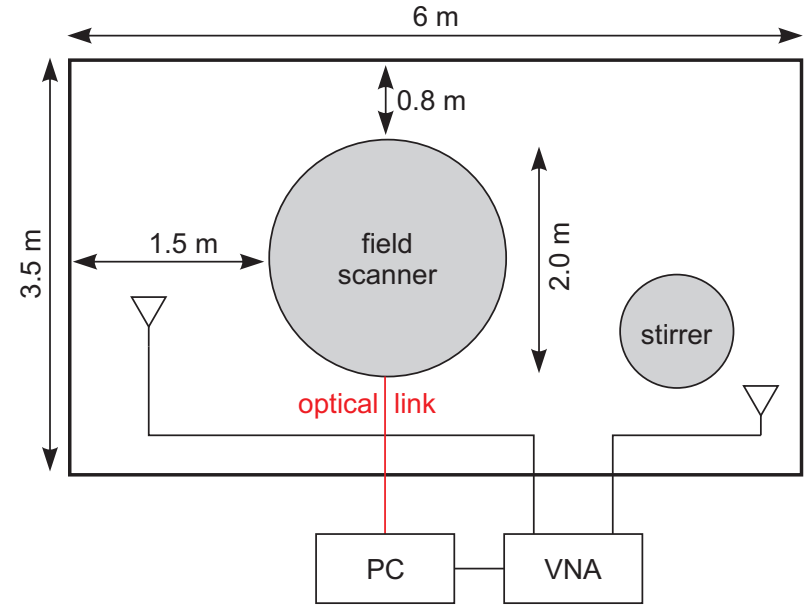

Fig. 5: Schematic representation (not to scale) of the setup employed in perturbation assessment.

two thin walls are almost half a wavelength apart at $3 \mathrm{GHz}$, explaining the constructive interference and the surge in the local reflectivity. Clearly, this last phenomenon is frequency dependent and must not be expected to be monotonous in frequency, as predicted by (4). Overall the arm is confirmed to be very weakly scattering, with a reflectivity well below $-20 \mathrm{~dB}$ up to $3 \mathrm{GHz}$.

\section{EXPERIMENTAL ASSESSMENT OF ROBOT-INDUCED PERTURBATIONS IN COMPLEX MEDIA}

Previous results suggest that the dielectric scanner can be regarded as a very weak scatterer. But this property can be hardly translated into a measure of perturbation of a medium where the robot is operated. In case of multiple scattering events, weak single-scattering interactions of waves with the robot can build up strongly enough to lead to non-negligible perturbations, as concluded in the next section. It is therefore important to introduce an experimental procedure to assess the degree of perturbation generated by moving the robot.

A reverberation chamber was considered as the basis for generating different complex media. Reverberation chambers are nowadays the standard test facility for analyzing the performance of wireless communications in realistic complex media. As shown in [3], by loading them even with very small volumes of absorbers their level of reverberation can be easily controlled and reduced, passing from a very large number of reflections, and thus propagation paths, to a more limited number, as likelier in real-life settings.

\section{A. Assessing the degree of coherence}

Suppose the probe holder is currently found at the position $r \in \Sigma$, in order to measure, e.g., the vector electric field $\boldsymbol{E}(\boldsymbol{r})$ generated by a source, e.g., an antenna. As the robot moves from one position to the next one, it behaves as a sort of ineffective field stirrer. For this reason, fields should be expressed as functions not only of the position $r$ where they are measured, but also of the position $s$ of the probe holder, i.e., $\boldsymbol{E}(\boldsymbol{r} ; \boldsymbol{s})$. The degree of coherence could then be

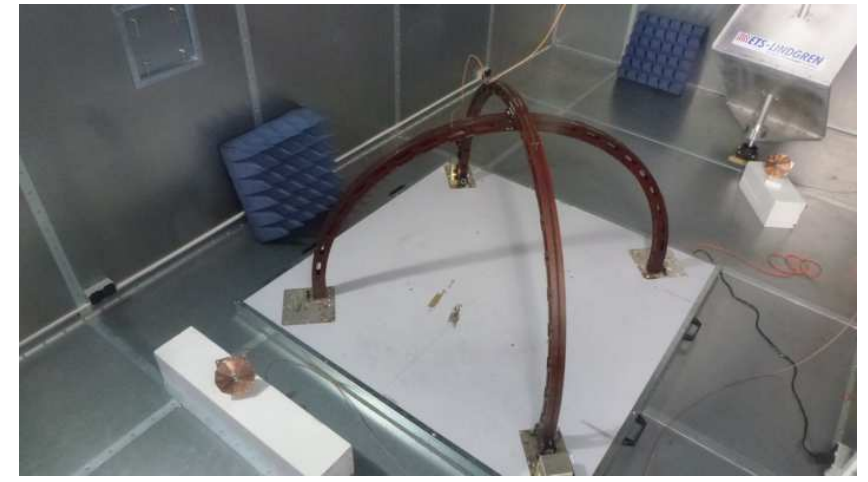

Fig. 6: The robot as tested in the reverberation chamber, with two of the absorbers used in order to control the spread time of the chamber.

assessed by monitoring how strongly $\boldsymbol{E}(\boldsymbol{r} ; \boldsymbol{s})$ is sensitive to $s$. An equivalent measure of perturbation, used hereafter, is the coherence of the medium as $s$ changes. This quantity is defined as the correlation coefficient between $\boldsymbol{E}(\boldsymbol{r} ; \boldsymbol{s})$, for a generic $s$, and $\boldsymbol{E}\left(\boldsymbol{r} ; \boldsymbol{s}_{o}\right)$, with $\boldsymbol{s}_{o}$ a position of the probe-holder serving as a reference.

In practice, testing a varying $r$ and $s$ at the same time is hardly feasible, as it would require a number of manual displacements over $\Sigma$ of a field probe, times the number of positions tested with the scanner. A simpler approach is possible, based on the use of an alternative test setup presented in Figs. 5 and 6. It requires the introduction of a receiving antenna, whose output voltages $V(s)$ can be demonstrated to be affected by the robot-induced perturbation as much as the field distribution to be measured over $\Sigma$. In other words, the receiving antenna is a proxy of the coherence of the field samples. This setup can also be understood as a direct measure of the perturbation in the Green functions (or transfer functions) of the medium, for a specific choice of transmitting and receiving antennas. It is then clearly necessary to formally demonstrate two points: 1) the position and type of antennas have no impact on the results and 2) the correlation computed from measured transfer functions is an accurate estimate of the correlation that would be observed for field samples. These two non-trivial properties are formally demonstrated in App. A and B, for the limit case of a diffused field, or Rayleigh propagation.

\section{B. Experimental setup}

The methodology discussed in the previous section was directly implemented in one of CentraleSupelec's reverberation chambers, a cuboid of $6 \times 3.5 \times 2.5 \mathrm{~m}^{3}$ containing the prototype of the robot described in Sec. III. It can be operated starting from about $300 \mathrm{MHz}$, where it can be regarded as sufficiently overmoded to ensure diffuse field conditions.

Two monocone antennas were used for the transmitting (source) and the receiving (proxy) antennas, positioned as shown in Fig. 5. From $400 \mathrm{MHz}$ to $6 \mathrm{GHz}$ they present an input reflection coefficient below $-10 \mathrm{~dB}$. Their radiation efficiency has no impact on the results, since they consist 

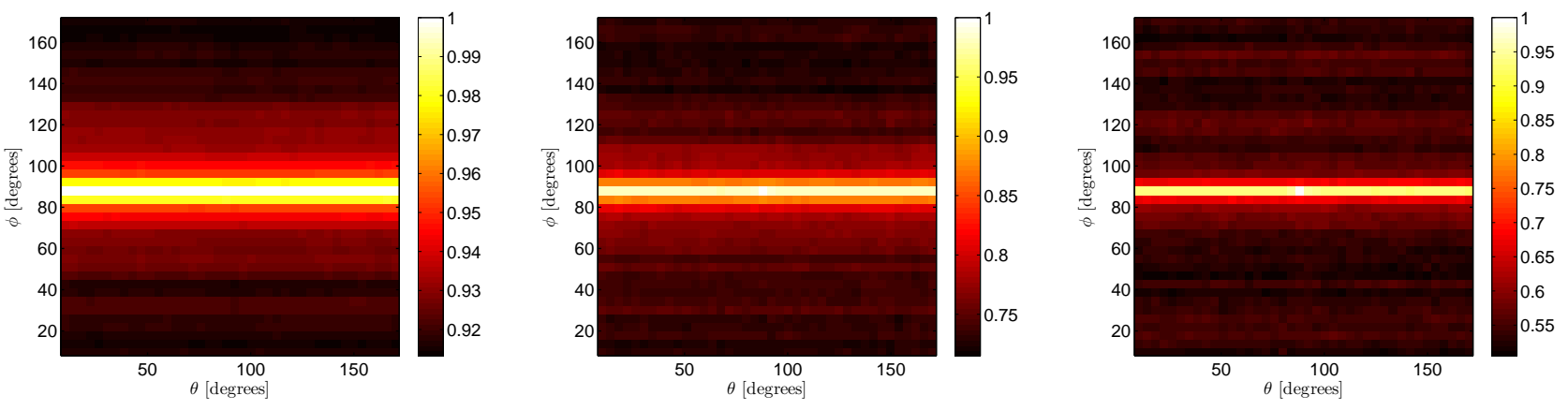

Fig. 7: Angular distribution of the correlation coefficient, estimated with respect to the transfer function for the probe holder stationing at $\theta=\varphi=90$ degrees. From left to right, results for 1,3 and $6 \mathrm{GHz}$, for a bandwidth of $200 \mathrm{MHz}$.

in correlation coefficients, thus energy normalized. Transfer functions between the antennas were measured by means of a vector network analyzer (Rohde \& Schwartz's ZVB8), calibrated by means of an automatic calibration kit, defining the ports of the antennas as reference planes.

Estimating correlation coefficients requires a population of random identically distributed samples. One possibility would have been to let the mechanical stirrer rotate, but it would have been a time-consuming solution. We rather borrowed the idea of frequency stirring [13], where a series of frequency samples are collected. An overall bandwidth of $200 \mathrm{MHz}$ was spanned, with 2000 uniformly distributed samples. Accessing the same number of samples with a mechanical stirrer would have required more than one hour, against the $5 \mathrm{~s}$ taken by the frequency sweep. Moreover, frequency samples are practically uncorrelated, whereas large sets of stirrer-generated samples are typically highly correlated thus yielding poor statistical properties.

This procedure was repeated for three central frequencies, namely 1, 3 and $6 \mathrm{GHz}$. The experimental protocol involved moving the probe holder to a given position, waiting $1 \mathrm{~s}$ to ensure a steady position and then measuring the $S_{21}$ over the three bandwidths. The probe holder was driven to scan a sector of the spherical surface, identified by $\theta, \varphi \in[10,170]$ degrees, over a $40 \times 40$ grid.

\section{RESUlts}

Following the approach described in Sec. V-A, the effect of the perturbation introduced by robot movements can be assessed by computing the correlation coefficient between the transfer functions obtained for the robot at a reference position $s_{o}$ and the entire set of transfer functions measured when the robot moves the probe holder at other positions $r \in \Sigma$. We start by choosing $s_{o}$ to have coordinates $\theta=\varphi=90$ degrees, i.e., the highest point reached by the scanner; the resulting correlation coefficient is shown in Fig. 7, as function of the position of the robot during the scanning phase, for three frequencies.

As the robot moves away from the reference position, the degree of coherence of the medium rapidly decreases before stabilizing about a practically constant background level, $\rho_{\infty}$. The total loss of coherence corresponding to the background
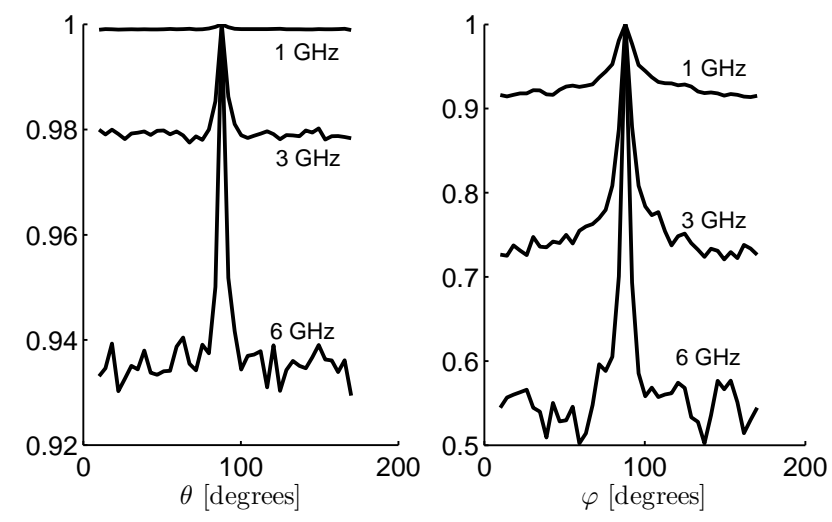

Fig. 8: $\varphi$ - and $\theta$-wise cuts of the correlation coefficients across the reference position.

level increases with the frequency, as expected from Secs. II and IV.

The results in Fig. 7 imply that the probe holder itself has a very minor impact on the overall correlation coefficient, resulting in almost unchanged results for $\theta$-wise scans, i.e., when the position of the mobile arm is unmodified, even at 6 $\mathrm{GHz}$; conversely, scans along $\varphi$ involve the displacement of the mobile arm, with its much larger surface and scattering cross-section. It is therefore possible to restrain our perturbation analysis on $\varphi$-wise scans. These observations can be better appreciated in Fig. 8, where $\theta$ - and $\varphi$-wise cuts of the results in Fig. 7 are shown, cutting across the reference position $\theta=\varphi=90$ degrees.

It is worth noticing how the correlation coefficients are practically insensitive to the direction along which the robot moves away from the reference position, leading to symmetric distributions of the correlation coefficient. This symmetry is not whatsoever due to any symmetry in the geometry of the chamber, since : (a) the robot is neither positioned nor oriented symmetrically with respect to the chamber walls; (b) the metallic stirrer, though not in use, ensures that all geometrical symmetry is broken.

In a more general fashion, the correlation coefficients appear to be underpinned by wide-sense stationary processes. Fig. 9 shows how, for a reference position shifting along $\varphi$, the 

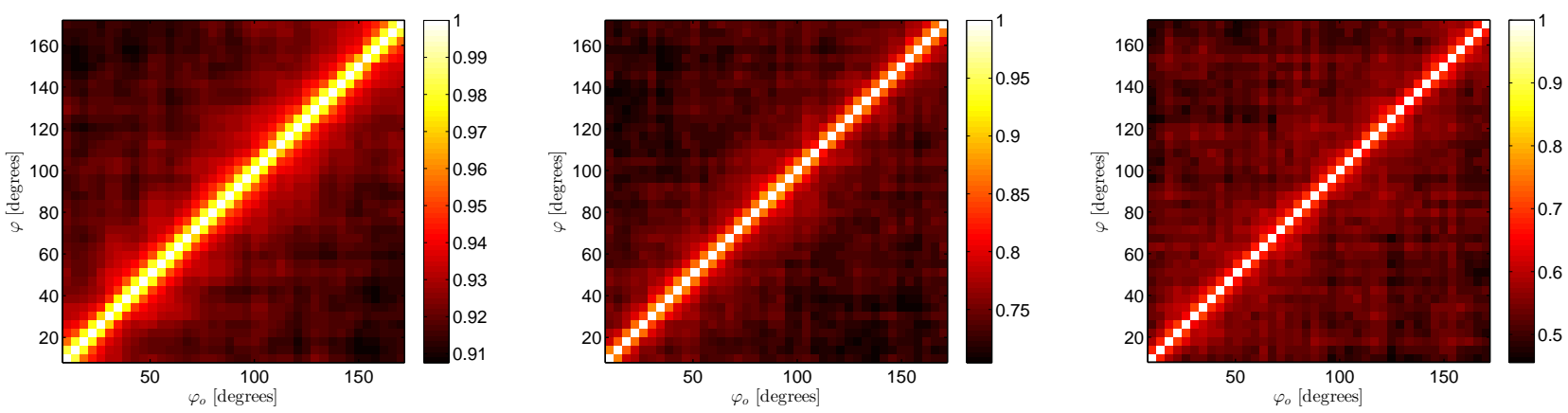

Fig. 9: Assessing stationarity in the perturbation: correlation coefficient for a varying reference position, across all positions along $\varphi$-wise scans. Results obtained for a $200 \mathrm{MHz}$ bandwidth, for a frequency equal to 1, 3 and $6 \mathrm{GHz}$.

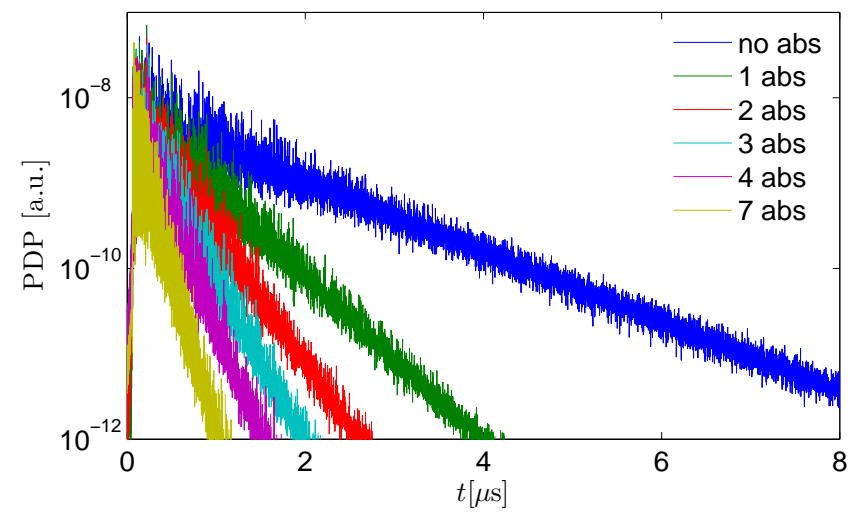

Fig. 10: Power-density profiles as estimated for an increasing number of absorbers at $6 \mathrm{GHz}$, for a $200 \mathrm{MHz}$ bandwidth.

entire set of correlation coefficients also appear to shift, with a remarkably stable reproduction of the same pattern. Symmetry is therefore just part of a more general trend.

All of the correlation patterns share the same features. In particular, the presence of a background correlation level $\rho_{\infty}$, weakly dependent on the distance between the rest position and the actual position of the mobile arm. The region displaying the strongest correlation shrinks as the frequency increases, as could be expected by recalling that as the frequency increases the level of perturbation of the mobile arm also increases (see Sec. II).

Another mechanism behind the perturbation needs to be acknowledged: depending on the level of reverberation of the medium, e.g., as measured by its average spread time $\bar{\tau}$ [14], waves propagating within the medium interact a varying number of times with the scanner, implying scattering events and thus perturbation. The overall perturbation can therefore be expected to be less severe for less complex propagation.

These conditions can be tested by including absorbers in the chamber, as those shown in Fig. 6. $\varphi$-wise scans were carried out again, for $\theta=90$ degrees, for a number of absorbers increasing from 1 to 4 and then 7 of them. Their impact on the reverberation of the chamber can be appreciated in Fig. 10 , where the power-delay profiles obtained from the transfer functions are shown, for a central frequency of $6 \mathrm{GHz}$ and a

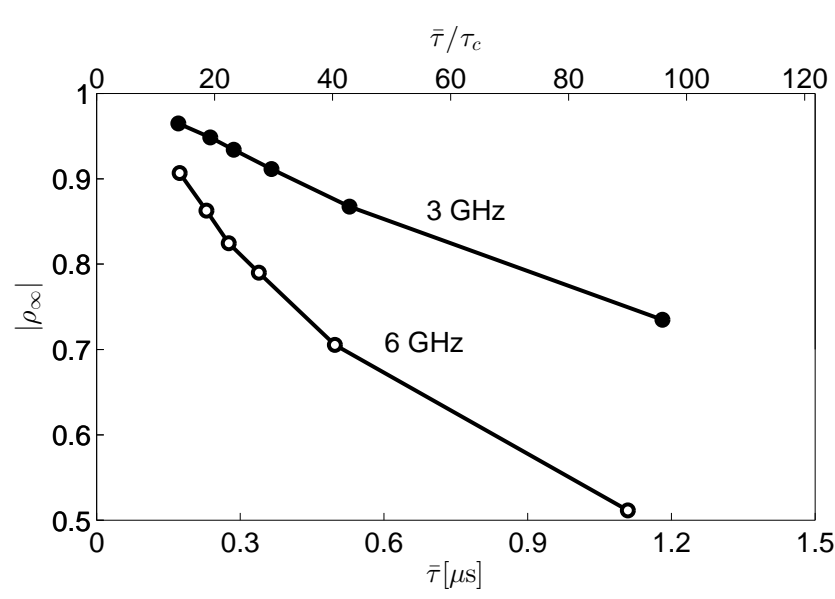

Fig. 11: Evolution of the background correlation $\rho_{\infty}$ as the medium average spread-time changes. The second (top) axis shows the approximate number of interactions with the scanner, assessed as $\bar{\tau} / \tau_{c}$.
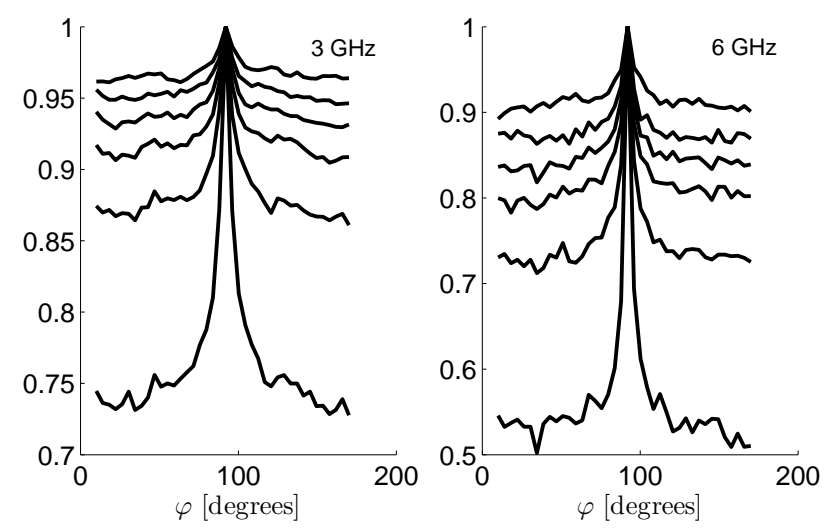

Fig. 12: Correlation for $\varphi$-wise cuts at 3 and $6 \mathrm{GHz}$, for an increasing number of absorbers, from 0 to 4 and for 7 of them (from bottom to top). 


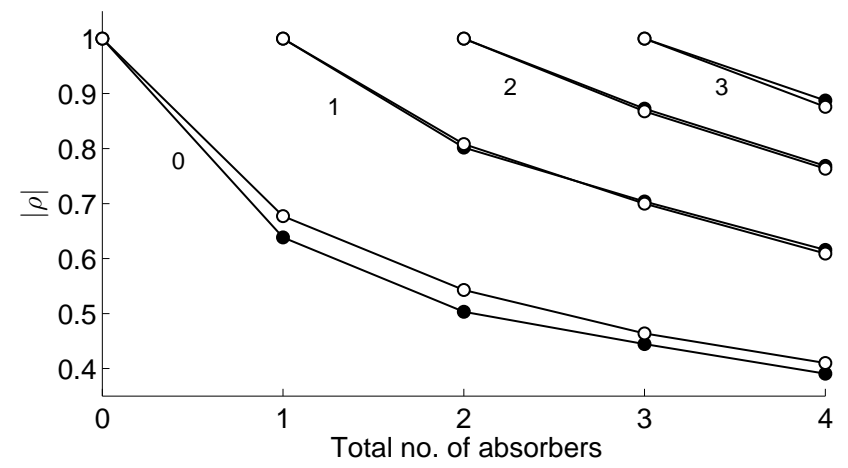

Fig. 14: Correlation coefficients for an increasing number of absorbers introduced into the chamber. Each curve stands for a different initial configuration, where a number of absorbers (indicated near to each curve) are initially present and to which others are subsequently added; each curve thus shows the amount of perturbation introduced by further loading the chamber. Black and white circles are for $3 \mathrm{GHz}$ and $6 \mathrm{GHz}$ data, respectively.

$500 \mathrm{MHz}$ bandwidth. From these results, $\bar{\tau}$ can be computed, serving as a basis for a rough estimate of the number of times waves interact with the scanner, given by $\bar{\tau} / \tau_{c}$, considering the characteristic time $\tau_{c}=V^{1 / 3} / c_{o}$, with $V$ the volume of the chamber. Fig. 11 confirms that as the time response of the chamber gets shorter, the perturbation introduced by the robot decreases. Notice how, even in the case of a heavily loaded chamber, the expected number of interactions is still high enough to consider the medium as complex.

Fig. 12 compares the perturbation introduced by the mobile arm of the scanner as it moves along $\varphi$, for an increasing number of absorbers. As $\bar{\tau}$ decreases, and the number of propagation paths within the chamber reduces, one could wonder if the stationarity observed in the perturbation is maintained; Fig. 13 proves that it still holds.

The results for the loaded chamber also shed some light into another issue: understanding how strongly a standard absorberbased scanner would modify the medium. For the position $\varphi=\theta=90$ degrees, the correlation between the transfer functions obtained with no absorber and for a varying number of absorbers was computed. The results of this operation are shown in Fig. 14, where for an initially empty chamber, the inclusion of a single absorber leads to a correlation around 60 $\%$, while two absorbers bring it around $50 \%$. For all these results, the frequency has a negligible impact, as could have been expected, since the absorbers have the same performance in this frequency range. Each curve represents the case of a chamber initially loaded with a number $n$ of absorbers, as additional absorbers are introduced: as could have been expected, for less complex media (i.e., already containing absorbers) the inclusion of further absorbers have a less severe effect on wave propagation. Only in the case of a heavily loaded chamber, e.g., with 3 absorbers, adding a further one has an acceptably low impact.

Since the surface covered by a single absorber is a fraction (roughly one tenth) of the total surface that would need to be covered if the robot were made out of metal, these results must be regarded as a low-bound estimate of the correlation loss.

\section{CONCLUSIONS}

The difficulties involved in measuring electromagnetic field distributions within complex media were discussed. Guidelines for designing a low-perturbation scanner were presented, together with a prototype. An indirect way of assessing the perturbation it introduces was theoretically discussed, monitoring the medium with a receiving antenna as a proxy.

The scanner was tested in a worst-case scenario, within a highly resonant reverberation chamber, showing levels of coherence higher than $90 \%$ below $1 \mathrm{GHz}$. Improved performance was demonstrated when the complexity of the propagation medium decreased, emulated by introducing losses. In this case the medium coherence was maintained above $90 \%$ even at $6 \mathrm{GHz}$.

The effectiveness of the proposed scanner is expected to make it possible to study empirically the intimate working of realistic media, with the perspective of validating more thoroughly propagation models and shedding some light on their behavior, without relying only on macroscopic metrics, as most often done due to the current lack of non-perturbative automatic solutions.

\section{ACKNOWLEDGEMENTS}

We acknowledge the fundamental contribution of Frédéric Duranton, owner and designer at Studioméca, who designed the robot. Stéphane Hopenzstand, counselor at Digiteo, is warmly thanked for his help and support throughout the AUTOTREC project.

\section{APPENDIX A}

\section{ESTIMATING COHERENCE FROM FIELD SAMPLES}

We first need, as a building block, to demonstrate that, given the nominal and perturbed electric fields measured at a position $\boldsymbol{r}$, their correlation is independent from $\boldsymbol{r}$. This goal can be achieved by expressing the electric field as a superposition of random plane waves [15],

$$
\boldsymbol{E}(\boldsymbol{r})=\int_{4 \pi} \mathrm{d} \hat{\boldsymbol{k}} \tilde{\boldsymbol{E}}(\hat{\boldsymbol{k}}) \mathrm{e}^{-\mathrm{j} k_{o} \hat{\boldsymbol{k}} \cdot \boldsymbol{r}},
$$

where $\tilde{\boldsymbol{E}}(\hat{\boldsymbol{k}})$ is the plane-wave spectrum (PWS) representation of $\boldsymbol{E}(\boldsymbol{r}), \hat{\boldsymbol{k}}$ is the direction of arrival of a single plane wave and $k_{o}$ is the wave number. Since several realizations can only be accessed by changing frequency (frequency stirring), frequency averages, indicated as $\langle\cdot\rangle_{\omega}$, are used instead of ensemble ones.

When the arm of the robot moves, the nominal PWS $\tilde{\boldsymbol{E}}_{o}(\hat{\boldsymbol{k}})$ gives place to

$$
\tilde{\boldsymbol{E}}(\hat{\boldsymbol{k}})=\rho \tilde{\boldsymbol{E}}_{o}(\hat{\boldsymbol{k}})+\delta \tilde{\boldsymbol{E}}(\hat{\boldsymbol{k}}),
$$

where $\left\langle\tilde{\boldsymbol{E}}_{o}^{*}(\hat{\boldsymbol{k}}) \cdot \delta \tilde{\boldsymbol{E}}(\hat{\boldsymbol{k}})\right\rangle_{\omega}=0$ by definition. These two random processes are assumed to be independent and must have zero average, as required for a diffuse field. 

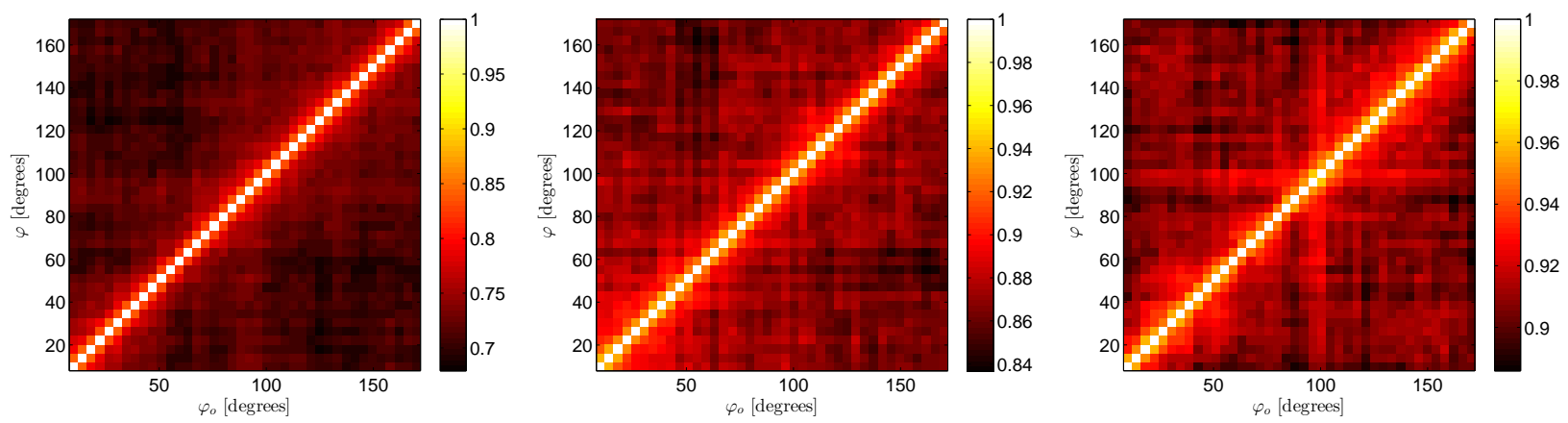

Fig. 13: Correlation coefficients computed in ordet to assess the stationarity of the perturbation of the mobile arm in a chamber loaded by 1,4 and 7 absorbers (from left to right), at $6 \mathrm{GHz}$.

The goal of the following derivation is twofold : first, to prove that correlation coefficients

$$
\hat{\rho}(\boldsymbol{r})=\left\langle\boldsymbol{E}_{o}^{*}(\boldsymbol{r}) \cdot \boldsymbol{E}(\boldsymbol{r})\right\rangle_{\omega} /\left\langle\left\|\boldsymbol{E}_{o}(\boldsymbol{r})\right\|^{2}\right\rangle_{\omega}
$$

are independent of the position $\boldsymbol{r}$; second, to show that $\hat{\rho}(\boldsymbol{r})=\rho$, i.e., that they are unbiased estimators of the perturbation introduced by the robot movement. Satisfying these two properties will imply that the perturbation introduced by the robot on the electric-field samples over $\Sigma$ can be assessed from data collected at another fixed position, if enough independent samples are collected. App. B builds on these properties.

We start by substituting (6) in the numerator of (8)

$$
\begin{aligned}
& \left\langle\boldsymbol{E}_{o}^{*}(\boldsymbol{r}) \cdot \boldsymbol{E}(\boldsymbol{r})\right\rangle_{\omega}= \\
& \quad \iint \mathrm{d} \hat{\boldsymbol{k}}_{1} \mathrm{~d} \hat{\boldsymbol{k}}_{2}\left\langle\tilde{\boldsymbol{E}}_{o}^{*}\left(\hat{\boldsymbol{k}}_{1}\right) \cdot \tilde{\boldsymbol{E}}\left(\hat{\boldsymbol{k}}_{2}\right)\right\rangle_{\omega} \mathrm{e}^{-\mathrm{j} k_{o} \boldsymbol{r} \cdot\left(\hat{\boldsymbol{k}}_{2}-\hat{\boldsymbol{k}}_{1}\right)}
\end{aligned}
$$

and, recalling the independence of $\tilde{\boldsymbol{E}}_{o}(\hat{\boldsymbol{k}})$ and $\delta \tilde{\boldsymbol{E}}(\hat{\boldsymbol{k}})$

$$
\left\langle\tilde{\boldsymbol{E}}_{o}^{*}\left(\hat{\boldsymbol{k}}_{1}\right) \cdot \delta \tilde{\boldsymbol{E}}\left(\hat{\boldsymbol{k}}_{2}\right)\right\rangle_{\omega}=\left\langle\tilde{\boldsymbol{E}}_{o}^{*}\left(\hat{\boldsymbol{k}}_{1}\right)\right\rangle_{\omega} \cdot\left\langle\delta \tilde{\boldsymbol{E}}\left(\hat{\boldsymbol{k}}_{2}\right)\right\rangle_{\omega}=0,
$$

hence

$$
\left\langle\tilde{\boldsymbol{E}}_{o}^{*}\left(\hat{\boldsymbol{k}}_{1}\right) \cdot \tilde{\boldsymbol{E}}\left(\hat{\boldsymbol{k}}_{2}\right)\right\rangle_{\omega}=\rho\left\langle\tilde{\boldsymbol{E}}_{o}^{*}\left(\hat{\boldsymbol{k}}_{1}\right) \cdot \tilde{\boldsymbol{E}}_{o}\left(\hat{\boldsymbol{k}}_{2}\right)\right\rangle_{\omega} .
$$

Substituting this result back into (9) and switching the order of integration we obtain

$$
\left\langle\boldsymbol{E}_{o}^{*}(\boldsymbol{r}) \cdot \boldsymbol{E}(\boldsymbol{r})\right\rangle_{\omega}=\rho\left\langle\left\|\boldsymbol{E}_{o}(\boldsymbol{r})\right\|^{2}\right\rangle_{\omega},
$$

and, ultimately,

$$
\hat{\boldsymbol{\rho}}(\boldsymbol{r})=\rho,
$$

a result holding for any $r$. Hence, the degree of perturbation introduced by changing the position of the robot can be measured everywhere in space, e.g., by a receiving antenna, starting from frequency-domain field measurements.

\section{APPENDIX B}

\section{EQUIVALENCE BETWEEN FIELD AND RECEIVED-VOLTAGE CORRELATIONS}

In practice, output voltages at the port of an antenna are often more accessible than field samples. It is therefore necessary to extend the result proven in the previous appendix to the case of voltage measurements. In particular, it is fundamental to prove that the type of antenna used has no impact on the estimate of the residual correlation.

Let us consider an antenna, found at the position $r$. Its output voltage is monitored before and after robot moves; these two voltages are referred to as $V_{o}(\boldsymbol{r})$ and $V(\boldsymbol{r})$, respectively.

In order to prove that

$$
\left\langle V_{o}^{*}(\boldsymbol{r}) V(\boldsymbol{r})\right\rangle_{\omega} /\left\langle\left|V_{o}(\boldsymbol{r})\right|^{2}\right\rangle_{\omega}=\rho,
$$

we express output voltages in terms of the PWS

$$
V(\boldsymbol{r})=\int_{4 \pi} \mathrm{d} \hat{\boldsymbol{k}} \boldsymbol{F}(\hat{\boldsymbol{k}}) \cdot \tilde{\boldsymbol{E}}(\hat{\boldsymbol{k}}) \mathrm{e}^{-\mathrm{j} k_{o} \hat{\boldsymbol{k}} \cdot \boldsymbol{r}},
$$

where $\boldsymbol{F}(\hat{\boldsymbol{k}})$ is the receiving pattern of the antenna [16]. Using this expression in the numerator of (14),

$$
\begin{aligned}
& \left\langle V_{o}^{*}(\boldsymbol{r}) V(\boldsymbol{r})\right\rangle_{\omega}= \\
& \iint \mathrm{d} \hat{\boldsymbol{k}}_{1} \mathrm{~d} \hat{\boldsymbol{k}}_{2} \boldsymbol{F}^{*}\left(\hat{\boldsymbol{k}}_{1}\right) \cdot \boldsymbol{D}\left(\hat{\boldsymbol{k}}_{1}, \hat{\boldsymbol{k}}_{2}\right) \cdot \boldsymbol{F}\left(\hat{\boldsymbol{k}}_{2}\right) \mathrm{e}^{-\mathrm{j} k_{o} \boldsymbol{r} \cdot\left(\hat{\boldsymbol{k}}_{2}-\hat{\boldsymbol{k}}_{1}\right),}
\end{aligned}
$$

where

$$
\boldsymbol{D}\left(\hat{\boldsymbol{k}}_{1}, \hat{\boldsymbol{k}}_{2}\right)=\left\langle\tilde{\boldsymbol{E}}_{o}^{*}\left(\hat{\boldsymbol{k}}_{1}\right) \tilde{\boldsymbol{E}}\left(\hat{\boldsymbol{k}}_{2}\right)\right\rangle_{\omega}
$$

is a dyadic function. Its individual terms $\left[\boldsymbol{D}\left(\hat{\boldsymbol{k}}_{1}, \hat{\boldsymbol{k}}_{2}\right)\right]_{i j}$ can be related to those of the electric-field PWS, e.g., $[\boldsymbol{E}(\hat{\boldsymbol{k}})]_{i}$, as

$$
\begin{aligned}
& {\left[\boldsymbol{D}\left(\hat{\boldsymbol{k}}_{1}, \hat{\boldsymbol{k}}_{2}\right)\right]_{i j}=\left\langle\left[\boldsymbol{E}_{o}^{*}\left(\hat{\boldsymbol{k}}_{1}\right)\right]_{i}\left[\boldsymbol{E}\left(\hat{\boldsymbol{k}}_{2}\right)\right]_{j}\right\rangle_{\omega}=} \\
& \rho\left\langle\left\|\boldsymbol{E}_{o}\left(\hat{\boldsymbol{k}}_{1}\right)\right\|^{2}\right\rangle_{\omega} \delta\left(\hat{\boldsymbol{k}}_{1}-\hat{\boldsymbol{k}}_{2}\right) \delta_{i j}
\end{aligned}
$$

thanks to (7), with $\delta\left(\hat{\boldsymbol{k}}_{1}-\hat{\boldsymbol{k}}_{2}\right)$ Dirac's delta distribution and $\delta_{i j}$ Kronecker's delta. This result is based on two properties postulated for diffuse media : 1) fields, and thus also PWSs, are unpolarized and their individual scalar components can be modeled as i.i.d. random processes; 2) their PWSs present no angular correlation.

Following (18), (16) simplifies into

$$
\left\langle V_{o}^{*}(\boldsymbol{r}) V(\boldsymbol{r})\right\rangle_{\omega}=\rho \tilde{E}_{o}^{2} \int \mathrm{d} \hat{\boldsymbol{k}}\|\boldsymbol{F}(\hat{\boldsymbol{k}})\|^{2},
$$

where

$$
\tilde{E}_{o}^{2}=\left\langle\left\|\boldsymbol{E}_{o}\left(\hat{\boldsymbol{k}}_{1}\right)\right\|^{2}\right\rangle_{\omega} \forall \hat{\boldsymbol{k}}
$$


is the average power of each plane-wave contributing to the PWS. The independence from the direction of arrival is a fundamental property postulated for diffusive media [17].

Following essentially the same procedure, it can be shown that

$$
\left\langle\left\|V_{o}(\boldsymbol{r})\right\|^{2}\right\rangle_{\omega}=\tilde{E}_{o}^{2} \int \mathrm{d} \hat{\boldsymbol{k}}\|\boldsymbol{F}(\hat{\boldsymbol{k}})\|^{2},
$$

thus validating (14) for any $\boldsymbol{F}(\hat{\boldsymbol{k}})$ and $\boldsymbol{r}$.

\section{REFERENCES}

[1] "IEEE standard test procedures for antennas," ANSI/IEEE Std 149-1979, 1979.

[2] "IEEE recommended practice for near-field antenna measurements," IEEE Std 1720-2012, pp. 1-102, Dec 2012.

[3] C. Holloway, D. Hill, J. Ladbury, P. Wilson, G. Koepke, and J. Coder, "On the use of reverberation chambers to simulate a Rician radio environment for the testing of wireless devices," IEEE Transactions on Antennas and Propagation, vol. 54, no. 11, pp. 3167 -3177, 2006.

[4] C. Patané Lötbäck, A. Skårbratt, and C. Ornelius, "Extending the reverberation chamber using a channel emulator for characterisation of over-the-air performance of multiple-input-multiple-output wireless devices," Science, Measurement Technology, IET, vol. 9, no. 5, pp. 555$562,2015$.

[5] H. Naus, "Statistical electromagnetics: Complex cavities," Electromagnetic Compatibility, IEEE Transactions on, vol. 50, no. 2, pp. 316-324, May 2008.

[6] R. J. Pirkl and K. A. Remley, "Experimental evaluation of the statistical isotropy of a reverberation chamber's plane-wave spectrum," IEEE Transactions on Electromagnetic Compatibility, vol. 56, no. 3, pp. 498 509, 2014.

[7] A. Ishimaru, Wave propagation and scattering in random media. WileyIEEE Press, 1999, vol. 12.

[8] L. Tsang and J. A. Kong, Scattering of electromagnetic waves, advanced topics. John Wiley \& Sons, 2004, vol. 26.

[9] R. E. Collin, Foundations for microwave engineering. McGraw-Hill, 1966.

[10] W. Burnside and K. Burgener, "High frequency scattering by a thin lossless dielectric slab," Antennas and Propagation, IEEE Transactions on, vol. 31, no. 1, pp. 104-110, Jan 1983.

[11] J. Gere and B. Goodno, Mechanics of materials. Nelson Education, 2012.

[12] A. Cozza and F. Duranton, "Device for characterizing electromagnetic waves," Patent application WO2 016128928,08 18, 2016.

[13] D. Hill, "Electronic mode stirring for reverberation chambers," Electromagnetic Compatibility, IEEE Transactions on, vol. 36, no. 4, pp. 294-299, Nov 1994.

[14] R. Vaughan and J. B. Andersen, Channels, propagation and antennas for mobile communications. Institution of Electrical Engineers, 2003.

[15] D. Hill, "Plane wave integral representation for fields in reverberation chambers," IEEE Transactions on Electromagnetic Compatibility, vol. 40, no. 3, pp. 209-217, 1998.

[16] R. Collins and F. Zucker, Antenna Theory. McGraw-Hill, New York, 1969.

[17] H. Kuttruff, Room acoustics. Taylor \& Francis, 2000. 